\title{
Efficacy of Silk Fibroin Based Bio-Ink in 3D Printing for Tissue Engineering
}

\author{
Manjunath RN* \\ Indian Institute of Technology Delhi, India \\ *Corresponding author: Manjunath RN, Indian Institute of Technology Delhi, Hauz Khas, New Delhi- 110016, India
}

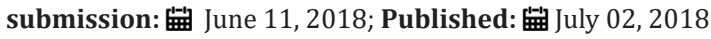

\section{Opinion}

\section{Backdrop}

Today, countless peoples are suffering and dying from various diseases and one of the reason being the non-compatibility and failure of life saving drugs. In fact 90 percent of the new drug that are tested in labs, on animals trials which are promised to cure the problems actually fail in certain humans. Sometimes, the drug failures during human trials are tragic enough to result in rapid multiple organ failures making the human tester incapable from leading his/her normal lifestyle [1]. The drug that demonstrated its ability to cure in the laboratories may not work same on all people as each human body is unique. This poses to be a major challenge for the drug manufacturers whose significant point of concern would be to develop safe and effective drug which is a risky, time consuming and highly expensive process in reality. In finding solutions to avoid such devastating drug failures and detrimental side effects on human trials, engineering and biotechnologists have come forward to work on developing human tissues outside the body and test the drugs on these tissues. As each individual are different from one another, the tissues developed form the body cells would be unique and testing the drugs on such tissues would accurately determine its safety, compatibility and effectiveness prior to human trials [2]. This can ease the process of drug development by reducing our reliance in animal trials and further increasing the acceptance rates with almost zero chance of rejection, ultimately ending up with better, safer and cheaper drugs.

\section{D bio-printing}

One of the means to achieve a clear-cut defined biomimetics implants for drug trials are by using the technique of 3D bio-printing. A3D bio-printer that functions with computer assisted approach and precise controls can provide a cutting-edge solution to facilitate the scientific and clinical objectives of producing biologically inspired 3D structures [3]. Particularly in the field of tissue engineering 3D bio-printing comes with several opportunities since it can allow the fabrication reproducibility and patient specific implants that can intimately mimic the native tissue characteristics with respect to structural performance, bio-degradability, bio-functionality and response to new drugs [4].
Though, the endless potential of 3D printing technique has gained much attention in today's bio-medical applications, there are several engineering problems and challenges that need to be tackled. Lack of ideal bio-inks with required bio-compatibility and printability has been recognized as a major barricade that has stalled the speedy and successful development of the 3D printing in printing complex tissues [5]. Most of the inks that are currently being used in 3D printing are based on silicones, collagen, alginates, gelatin etc. These inks are sensitive to temperature, $\mathrm{pH}$ changes and lack structural stability that requires cross-linking additives to toughen these materials. Inclusion of such additives to the bio-ink can damage the body cells and other biological components that the researchers want to develop [6]. Therefore, the pursuit of a novel bio-ink material that can potentially be used in development of biological implants in which the success of actual tissue engineering lies upon remains a challenge.

\section{Silk fibroin based bio-ink for 3D printing}

Fibroin which is the chief constituent of silk has gained a major attention in the field of scaffold development and tissue engineering for decades. Silk fibroin is a natural and easily available protein associated with excellent mechanical strength, bio-compatibility, bio-degradability and options for flexible structural modification that holds almost all the requirements for an ideal material for bioprinting $[7,8]$. The process of fibroin based bio-ink development primarily begins with the extraction and purification of fibroin from the naturally available silk filaments that can be performed with variety of aqueous or solvent based processing techniques [9]. Further, they are mixed suitable additives that are necessary to provide structural support for soft tissue construction especially for larger constructs [10].

Compaan et al. [11] proposed a two-step gelation process using alginate as a sacrificial hydrogel during ink-jetting process and calcium alginate to achieve rapid gelation during printing process. This technique enabled the successful bio-printing of fibroin constructs which suitable for long term culture [11]. Jose et al., introduced a fibroin based biocompatible and two-part room 
temperature curing bio-ink that were physically stabilised by the addition of polyols and glycerine that could impart structural robustness to the prints [6]. In other work, Rodriguez et al., incorporated gelatin as bulking agent and glycerol as a non-toxic additive to develop a material system with optimal rheological properties suitable for extrusion and solidification upon deposition. Bio-ink formulations were also optimised by modulating the concentration of fibroin, gelatine and glycerol to demonstrate a stable material under physiological conditions that can be tuned to match required soft tissue characteristics [12]. An extensive search of research work done till date would prove that silk fibroin can be a great promise and boon for the tissue engineering especially when the concept of bio-ink and 3D bio-printing concern. In addition to bio-compatibility, stability and printability 3D printed silk structures are relatively cheaper than those constructed from the synthetic polymer systems [13].

\section{Outlook}

India being the second the second largest producer of silk globally is gifted with easy availability of the resources needed for 3D printing. An attempt to explore the endless possibilities of bioprinting and silk based bio-compatible structural implants has to be made further with prime focus of commercialisation. This would not only strengthen the scientific expansion in the medical sector reducing but also fortify Indian economy by boosting its sericulture industry.

\section{References}

1. https://www.genengnews.com/the-lists/unlucky-13-top-clinical-trialfailures-of-2017/77901028

2. (2018) Formation of human tissue to improve drug testing and reduce animal research
3. Cengiz IF, Pitikakis M, Cesario L, Parascandolo P, Vosilla L, et al. (2016) Building the basis for patient-specific meniscal scaffolds: from human knee MRI to fabrication of 3D printed scaffolds. Bioprinting 1: 1-10.

4. Kim SH, Yeon YK, Lee JM, Chao JR, Lee YJ, et al. (2018) Precisely printable and biocompatible silk fibroin bioink for digital light processing 3D printing. Nature communications 9(1): 1620.

5. Costa JB, Silva-Correia J, Oliveira JM, Reis RL (2017) Fast setting silk fibroin bioink for bioprinting of patient-specific memory-shape implants. Adv Health Mater 6(22) doi: 10.1002/adhm.201701021.

6. Jose RR, Brown JE, Polido KE, Omenetto FG, Kaplan DL (2015) Polyol-silk bioink formulations as two-part room-temperature curable materials for 3D printing. ACS Biomaterials Science \& Engineering 1(9): 780-788.

7. Sommer MR, Schaffner M, Carnelli D, Studart AR (2016) 3D printing of hierarchical silk fibroin structures. ACS Applied Materials \& Interfaces 8(50): 34677-34685

8. Rider P, Zhang $\mathrm{Y}$, Tse C, Zhang $\mathrm{Y}$, Jayawardane D, Stringer J, et al. (2016) Biocompatible silk fibroin scaffold prepared by reactive inkjet printing. Journal of Materials Science 51(18): 8625-8630.

9. Vepari C, Kaplan DL (2007) Silk as a biomaterial. Prog Polym Sci 32(8-9): 991-1007.

10. Rockwood DN, Preda RC, Yücel T, Wang X, Lovett ML, et al. (2011) Materials fabrication from Bombyx mori silk fibroin. Nat protoc 6(10): 1612.

11. Compaan AM, Christensen K, Huang Y (2016) Inkjet bioprinting of 3D silk fibroin cellular constructs using sacrificial alginate. ACS Biomaterials Science \& Engineering 3(8): 1519-1526.

12. Rodriguez MJ, Brown J, Giordano J, Lin SJ, Omenetto FG, et al. (2017) Silk based bioinks for soft tissue reconstruction using 3-dimensional (3D) printing with in vitro and in vivo assessments. Biomaterials 117: 105115.

13. Benedict (2016) Korean researchers develop new silk 3D printing system for bone fracture implants.
Creative Commons Attribution 4.0 International License

For possible submissions Click Here

\section{Submit Article}

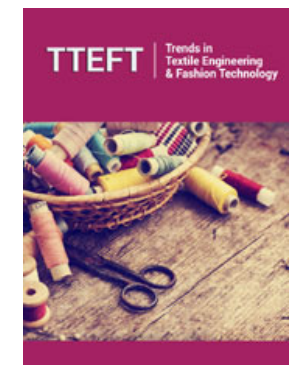

Trends in Textile Engineering \& Fashion Technology

\section{Benefits of Publishing with us}

- High-level peer review and editorial services

- Freely accessible online immediately upon publication

- Authors retain the copyright to their work

- Licensing it under a Creative Commons license

- Visibility through different online platforms 